\title{
RESEARCH NOTES
}

\section{A CHARACTERIZATION OF PSEUDOCOMPACTNESS}

\section{PRABDUH RAM MISRA}

\author{
I.M.F. - U.F.G. \\ Caixa Postal - 597 \\ 74000 - Goiânia - Go, BRASIL \\ and \\ VINODKUMAR \\ I.I.T. \\ New Delhi, INDIA \\ (Received October 6, 1980)
}

ABSTRACT. It is proved here that a completely regular Hausdorff space $\mathrm{X}$ is pseudocompact if and only if for any continuous function $f$ from $X$ to a pseudocompact space (or a compact space) $Y, f^{*} \phi$ is $z$-ultrafilter whenever $\phi$ is a z-ultrafilter on $\mathrm{X}$.

KEY WOROS AND PHRASES. Pseudocompact, BX, z-filter, z-ultra function. 1980 MATHEMATICS SUBJECT CLASSIFICATION CODES. Primary 54099.

1. INTRODUCTION.

For notations and basic results one is referred to [1]. We only consider here completely regular Hausdorff spaces.

Let $f$ be continuous from $X$ to $Y$. Let $\phi$ be a $z$-ultrafilter on $X$, then $f^{*} \phi$ denotes the $z$-filter $\left\{B \in Z(Y): f^{-1}(B) \varepsilon \phi\right\}$ on $Y$ and is known to be prime. We further know that a prime z-filter is contained in a unique z-ultrafilter. Let $\Delta(f) \phi$ denote the $z$-ultrafilter containing $f^{*} \phi$. Thus we have a function $\Delta(f)$ from $\beta X$ to $\beta Y$ sending $\phi$ to $\Delta(f) \phi$. The function $f$ is called $z$-ultra if $f_{\phi}^{*}=$ $\Delta(f) \phi$ for every z-ultrafilter $\phi$ on $\mathrm{X}$. 


\section{MAIN RESULTS}

PROPOSITION. A continuous function $f$ from $X$ to $Y$ is z-ultra if and only if for every zero-set $B$ in $Y, \Delta(f)^{-1}\left(\bar{B}^{\beta Y}\right)=\overline{f^{-1}(B)}$.

PROOF. Let $f$ be z-ultra. Then, $\phi \varepsilon \Delta(f)^{-1}\left(\vec{B}^{\beta Y}\right)$ if and only if $\Delta(f) \phi=$ $\mathrm{f}^{*} \phi \varepsilon \overline{\mathrm{B}}^{\beta Y}$. But this is equivalent to $B \in \mathrm{f}^{*} \phi$ or to $\mathrm{f}^{-1}(B) \varepsilon \phi$, which happens if and only if $\phi \in \overline{f^{-1}(B)}$.

Conversely, $B \in \mathrm{f}^{\star} \phi$ if and only if $\phi \varepsilon \overline{\mathrm{f}}^{-1}(\mathrm{~B})$, 1.e. $\Delta(\mathrm{f}) \phi \varepsilon \overline{\mathrm{B}}^{\beta Y}$, since ${\overline{f^{-1}(B)}}^{-\beta X}=\Delta(f)^{-1}\left(\bar{B}^{\beta Y}\right)$. But $\Delta(f) \phi \varepsilon \bar{B}^{\beta Y}$ is equivalent to saying that $B \varepsilon \Delta(f) \phi$. We see that $f^{*} \phi=\Delta(f) \phi$.

In order to prove the main theorem of the paper we need the following observations for pseudocompact spaces. If $\mathrm{X}$ is pseudocompact, then a subset of $\beta X$ is a zero-set if and only if it is closure of a zero-set in $X$ and conversely, a subset of $X$ is a zero-set in $X$ if and only if its closure is so in $\beta X$.

THEOREM. If a space $X$ is pseudocompact then any continuous function $f$ from $X$ to any pseudocompact space $Y$ is z-ultra. Conversely, if the inclusion of $X$ in $B X$ is $z$-ultra, then $X$ is pseudocompact.

$\underline{P R O O F}$. Let $B$ be a zero-set in $Y$. Since $\bar{B}^{B Y}$ is a zero-set in $B Y$ as $Y$ is pseudocompact, $\Delta(f)^{-1}\left(\bar{B}^{\beta Y}\right)$ is a zero-set in $\beta X$. Pseudocompactness of $X$ implies that $\Delta(f)^{-1}\left(\vec{B}^{\beta Y}\right)=\vec{A}^{\beta X}$ for some zero-set $A$ in $X$. We show that $A=f^{-1}(B)$. Since $\Delta(f) / X=f$, we observe that $\Delta(f)^{-1}(B) \cap X=f^{-1}(B)$. Clearly, $\Delta(f)^{-1}$ $\left(\vec{B}^{\beta Y}\right) \cap \quad x=\Delta(f)^{-1}(B) \cap \quad x=f^{-1}(B) . \quad N e x t, \Delta(f)^{-1}\left(\bar{B}^{\beta Y}\right) \Gamma_{1} x=\vec{A}^{\beta X} \cap$ $X=A$.. Hence $f^{-1}(B)=A$, and we have $f$ to be z-ultra.

Conversely, let 1 be the inclusion of $\mathrm{X}$ in $\beta \mathrm{X}$. Since $\Delta(1) / \mathrm{X}=1, \Delta(1)$ is the identity on $\beta X$. Let $B$ be a nonempty zero-set in $\beta X$. Since 1 is z-ultra, from the above proposition we have that $B=\Delta(1)^{-1}(B)=1^{-1}(B)=B \cap X^{B X}$ and $[1,6 I .1]$ shows that $X$ is pseudocompact.

As an application of our theorem we prove the following well known theorem due to Glicksberg [2]. 
THEOREM. If $\mathrm{X}$ is pseudocompact and $\mathrm{Y}$ is compact, then $\mathrm{X} \times \mathrm{Y}$ is pseudocompact.

PROOF. Let $\mathrm{f}: \mathrm{X} \times \mathrm{Y} \rightarrow \mathrm{Z}$ be a continuous function, $\mathrm{Z}$ some pseudocompact space. Consider a z-ultrafilter $\phi$ on $\mathrm{X} \times \mathrm{Y}$. Let $\pi_{2}$ : $\mathrm{X} \times \mathrm{Y} \rightarrow \mathrm{Y}$ denote the projection on the second coordinate. Since $Y$ is compact and $\pi^{*}{ }_{2}{ }^{\phi}$ is a $z$-filter, it is fixed as we11. Let $y_{0} \varepsilon \cap \pi^{*}{ }_{2} \phi$. Hence $\phi_{1}$, the restriction of $\phi$ to the subspace $x \times\left\{y_{0}\right\}$ is a z-ultrafilter on $x$ x $\left\{y_{0}\right\}$. Let $f_{1}$ denote the restriction of $f$ to the subspace $x \times\left\{y_{0}\right\}$. Since $X$ is pseudocompact, $f_{1}$ is $z-u l t r a$. Clearly, $\mathrm{f}^{*} \phi \subseteq \mathrm{f}_{1}^{*}{ }_{1}$. Next, let $\mathrm{B} \varepsilon \mathrm{f}_{1}^{*} \phi_{1}$. Hence $\mathrm{f}_{1}^{-1}(\mathrm{~B}) \varepsilon \phi_{1}$. Since $\mathrm{f}^{-1}$ (B) contains $f_{1}^{-1}(B), f^{-1}(B)$ intersects every member of $\phi$. Thus $f^{-1}(B) \varepsilon \phi$ as it is a z-ultrafilter. We get that $B \in \mathrm{f}^{*} \phi$. Hence $\mathrm{f}^{*} \phi=\mathrm{f}^{*}{ }_{1}{ }_{1}$ and it follows that $\mathrm{f}$ is $\mathrm{z}-$ ultra.

\section{ACKNOWLEDGEMENT}

This work was done while the first author was visiting Mehta Research Institute, Allahabad in summer 1977.

\section{REFERENCES}

1. Gillman, L. and Jerison, M., Rings of Continuous Functions. Van Nostrand, Princeton, 1960.

2. Glicksberg, I., Stone-Cech Compactifications of Products, Trans. Amer. Math. Soc. 90 (1959), 369-382. 


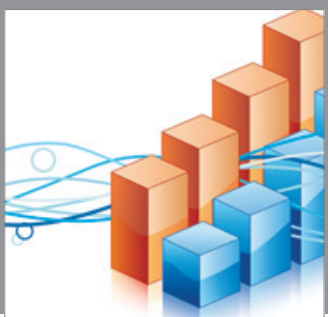

Advances in

Operations Research

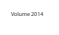

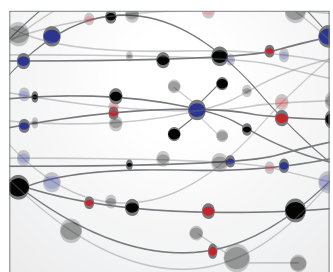

\section{The Scientific} World Journal
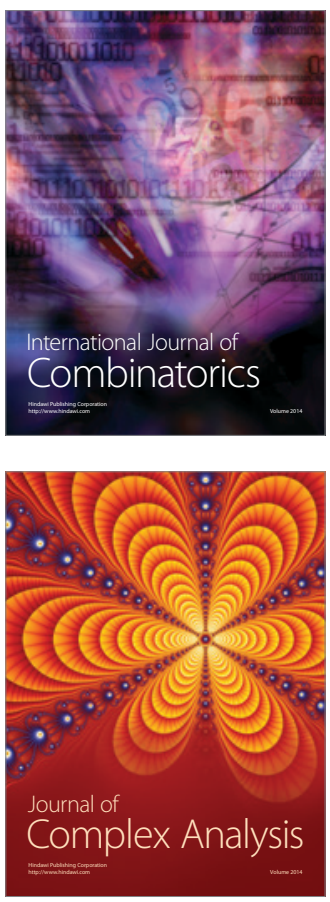

International Journal of

Mathematics and

Mathematical

Sciences
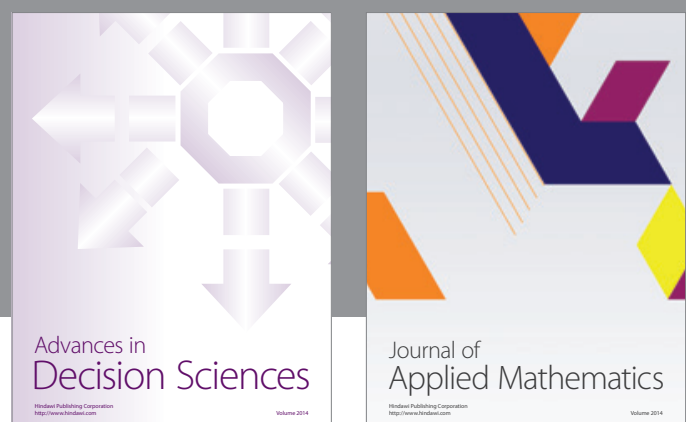

Journal of

Applied Mathematics
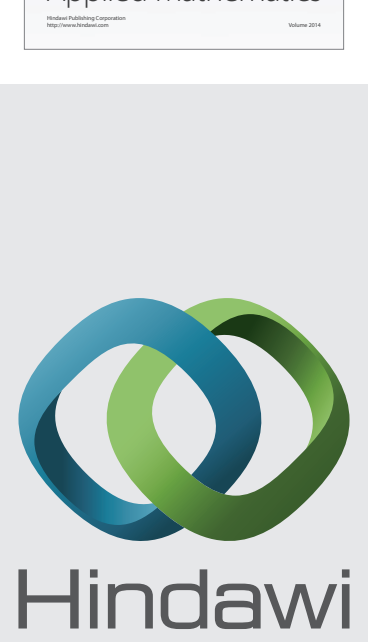

Submit your manuscripts at http://www.hindawi.com
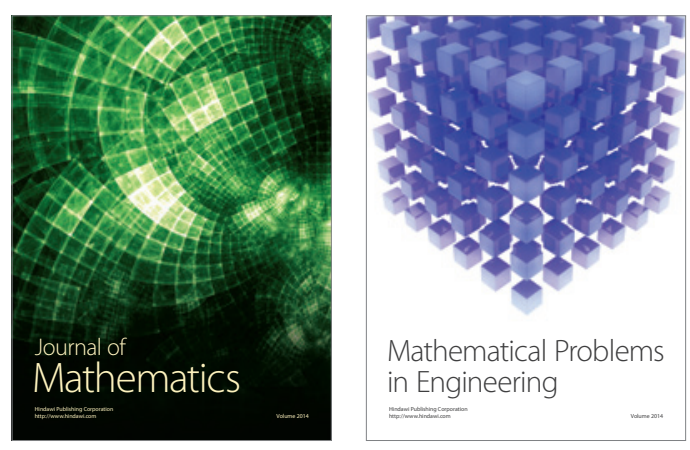

Mathematical Problems in Engineering
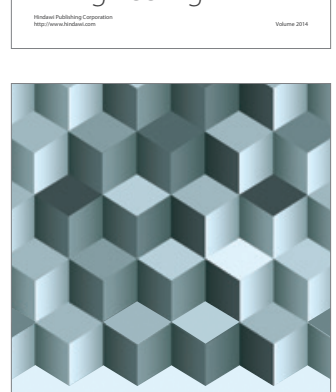

Journal of

Function Spaces
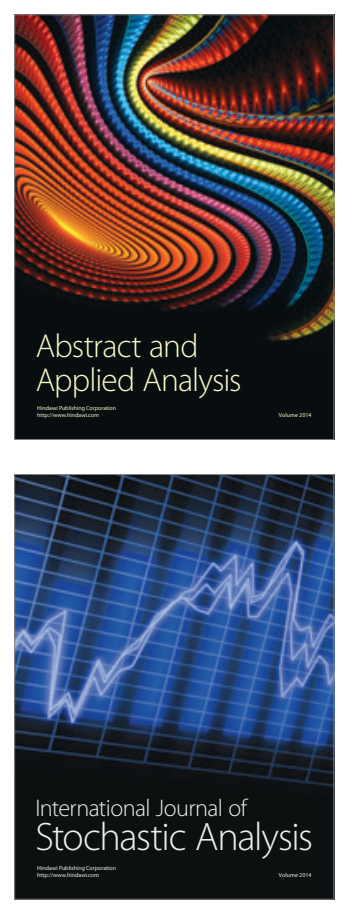

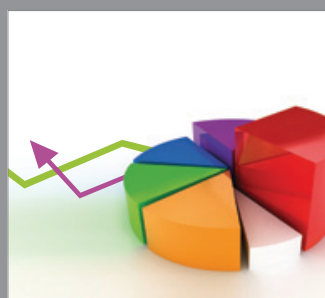

ournal of

Probability and Statistics

Promensencen
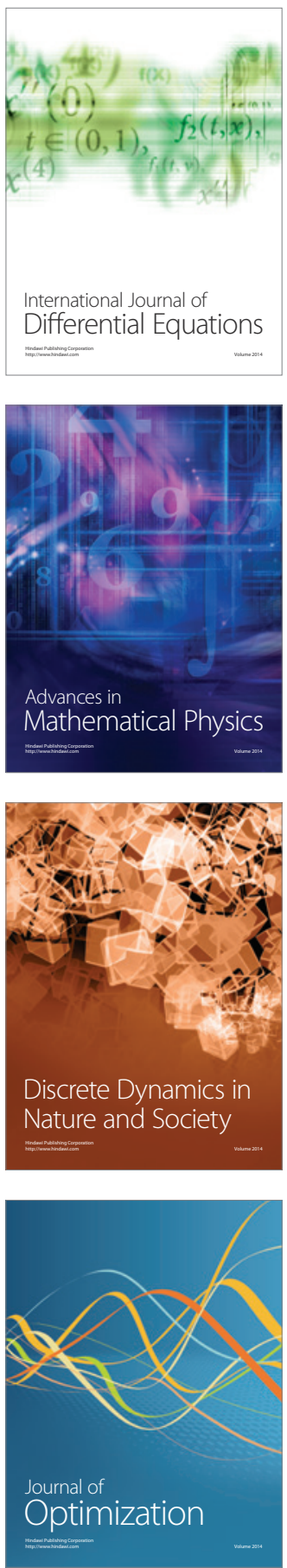